\title{
Benefiting wireless power transfer scheme in power domain based multiple access: ergodic rate performance evaluation
}

\author{
Anh-Tu Le ${ }^{1}$, Minh-Sang Van Nguyen ${ }^{2}$, Dinh-Thuan Do ${ }^{3}$ \\ ${ }^{1,2}$ Faculty of Electronics Technology, Industrial University of Ho Chi Minh City, Vietnam \\ ${ }^{3}$ Wireless Communications Research Group, Faculty of Electrical \& Electronics Engineering, Ton Duc Thang University, \\ Ho Chi Minh City, Vietnam
}

\section{Article Info}

Article history:

Received Aug 11, 2020

Revised Nov 7, 2021

Accepted Dec 11, 2021

\section{Keywords:}

Non-orthogonal multiple access

Ergodic capacity

Wireless power transfer

\begin{abstract}
Power domain based multiple access scheme is introduced in this paper, namely Nonorthogonal multiple-access (NOMA). We deploy a wireless network using NOMA together with a wireless power transfer (WPT) scheme for dedicated user over Nakagami- $m$ fading channel. When combined, these promising techniques (NOMA and WPT) improve the system performance in term of ergodic performance at reasonable coefficient of harvested power. However, fixed power allocation factors for each NOMA user can be adjusted at the base station and it further provide performance improvement. We design a new signal frame to deploy a NOMA scheme in WPT which adopts a linear energy harvesting model. The ergodic capacity in such a NOMA network and power allocation factors can be updated frequently in order to achieve a fair distribution among NOMA users. The exact expressions of ergodic capacity for each user is derived. The simulation results show that an agreement between analytic performance and Monte-Carlo simulation can be achieved.
\end{abstract}

This is an open access article under the CC BY-SA license.

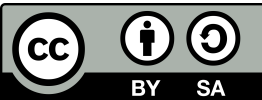

\section{Corresponding Author:}

Dinh-Thuan Do,

Wireless Communications Research Group,

Faculty of Electrical \& Electronics Engineering,

Ton Duc Thang University, Ho Chi Minh City, Vietnam.

Email: dodinhthuan@tdtu.edu.vn

\section{INTRODUCTION}

Recently, multiple access has raised much interest. A multiple access can be performed on a power domain with different power allocations for individual users. It can be considered a novel scheme since the non-orthogonal multiple access (NOMA) is considered a promising technique to improve the spectral efficiency (SE) of the upcoming fifth generation $(5 \mathrm{G})$ network [1,2]. NOMA and wireless power transfers are put together in order to satisfy the requirement for massive connections such as the Internet of Things (IoT) to support massive users [3]. When analysing the advantages of NOMA, an interesting idea is to realize that the multiple access in the power domain allows multiple users to be served at the same time at different levels of the assigned power resource. To achieve a better system performance compared with the conventional orthogonal multiple access (OMA) scheme, we apply power-domain multiplexing at the transmitter for the mixed signal. Subsequently, a successive interference cancellation (SIC) is required to separate the signal at the receivers [4]-[6]. 
In a distributed wireless network, such as for instance in Internet of Things (IoT) containing mobile devices, power supply should remain continuous yet the amount of energy is small [7, 8]. In such cases, a limited energy supply can be charged from the received signal through RF signal. For certain IoT applications, it is hard to replace the battery and/or there is no power line, it meets limited performance improvement. Fortunately, an energy harvesting technique has been proposed to help the limited power-aware devices harvest energy from the surrounding environment. To address any shortage problems of power supplied into numerous devices, various energy harvesting schemes have been introduced. Recently, radio frequency energy harvesting (RF-EH) have been recommended to be employed in a wireless network, based on the green communication concept. Such efficient solution of RF-EH is used to prolong the lifetime of energy-constraint devices in wireless communication systems [9]. Radio frequency (RF) energy harvesting [10], provides an improved performance in poor condition of power supply. The EH-based system models can provide flexible, sustainable and stable energy supply and have been widely tested in many scenarios of relaying networks [11]-[13].

In a different line of research, the idea of energy harvesting is introduced by deploying the NOMA system to overcome the shortage of the energy constraint devices in [15]-[18]. In these systems, the near users play the role of EH-based relays helping the far users while the main metric is considered, i.e. the outage performance is analyzed with varying parameters in several scenarios. In [18], a NOMA scheme was incorporated in the wireless powered networks. In this study, the authors presented an optimal resource allocation strategy. In addition, three opportunistic user selection schemes were examined[19], introducing a novel cooperative EH based NOMA protocol. In other works [19, 20], the authors considered the outage performance of many users in the NOMA system with the ability of EH based transmission, and a reasonable performance of proposed scheme is highlighted. However, the work in [20] is based on the ideal linear EH model.

However, secure resource allocation schemes are investigated in [21]-[23] with the results proving the advantages of NOMA systems. In a EH based NOMA system, the EH requirement needs to be considered. Moreover, certain challenges remain to be addressed in this system, especially where a practical non-linear EH model has been adopted. The main issue is how to assign the most favorable power allocation factors to each of NOMA users. Optimal outage or throughput performance in such NOMAs can be analysed, yet intractable forms of expressions relate to these metrics.

Unlike in [14]-[23], in this work, we focus on the new NOMA scheme, employing a wireless power transfer to exhibit the feedback of power allocation factors in NOMA to the base station (BS) in a wireless system. Until now, it had been mostly assumed that fixed power allocation factors applied to each NOMA user. However, the assignment plays a crucial role in achieving an improved performance. The feedback for these factors in NOMA is required in real applications and this issue has not been solved satisfactorily yet. It is worth mentioning that the limited power in massive users who require to be served at same time poses a serious problem. These discussions motivated us to look for a novel scheme. In the proposed scheme, the EH NOMA scheme allows to prioritize users, and subsequently find ergodic capacity to employ many applications in wireless communications.

The main contributions of this paper can be shown as:

- In this paper, we aim to satisfy the fairness in a resource allocation in NOMA, we provide information related to power allocation factor in uplink and then the performance of downlink in NOMA is studied.

- Energy harvesting is deployed in NOMA to help mobile users reuse the harvested power to provide signal transmission in uplink. Time splitting factors and power splitting factors are considered to achieve an improvement as NOMA and wireless power transfer are implemented.

- We characterize the system performance by exploiting the ergodic capacity. The exact expressions for the achievable ergodic rate of individual users are introduced. Further, we compare the performance of NOMA and OMA.

- The numerical results are shown to check the correctness of our proposed metric and to demonstrate the fairness enhancement of the proposed EH NOMA scheme.

The rest of this paper can be summarized as follows: Section II introduces the system model in case the energy harvesting protocol has been deployed in NOMA to perform the transmission in a wireless sensor system. In this section, the key computations such as expressions of SNR are investigated. In Section III, based on obtained SNR, we derive the analytic expressions of ergodic rate in delay-tolerant transmission. Section IV demonstrates the simulation results. Finally, Section V completes with the conclusion remarks for the paper and reviews the important results. 


\section{SYSTEM MODEL AND SINR COMPUTATIONS}

\subsection{Energy harvesting transmission model in NOMA}

This system model deploys a time-splitting relaying mode to perform the wireless charge for the mobile users who suffer limited power input. In the first phase, to employ downlink for the NOMA transmission, the base station (BS) sends a superimposed NOMA signal $\left(x_{U_{1}} a_{U_{1}}+x_{U_{2}} a_{U_{2}}\right)$ to $U_{1}$ and $U_{2}$ with fixed transmit power coefficients $a_{i}$, where $i \in\left\{U_{1}, U_{2}\right\}$. Power allocation factors are required to frequently updated in uplink and these dynamic factors satisfy $a_{U_{1}}^{2}+a_{U_{2}}^{2}=1$. We call $d_{i}^{l_{e}}$ is related to distance $d_{i}$ and $l_{e}$ is pathless exponent. To represent for the wireless channel between the BS and each user, we denote $h_{S i}$ as the Rayleigh channel model. $P_{S}$ stands for transmit power at the BS and in this case such power is divided into two parts: the first part $P_{S} \beta_{e}$ for a wireless power transfer while the second part for information processing $P_{S}\left(1-\beta_{e}\right)$. $\beta_{e}$ is the percentage of the harvested power, i.e. $0<\beta_{e}<1$. The mobile nodes are able to collect wireless power while requiring only a finite amount of energy for their information transmission to provide feedback of power allocation factors to the BS in uplink. This advantage of energy harvesting can be exploited for the small devices requiring only a small amount of energy. To facilitate the NOMA with the energy harvesting capability, the whole transmission block $T$ needs to be divided into two time slots.

For this study, we have designed the following frame of signal transmission. The first part of the frame serves to update the power allocation factors based on which the allocation to individual NOMA users is performed. To the best of authors' knowledge, a novel frame for NOMA had first been designed. It is worth noting that the key second part of the frame for the downlink transmission from the BS to two NOMA users using dynamic power allocation factors had been already known in the first part of the considered frame of the signal. System model of NOMA with wireless power transfer as shown in Figure 1.

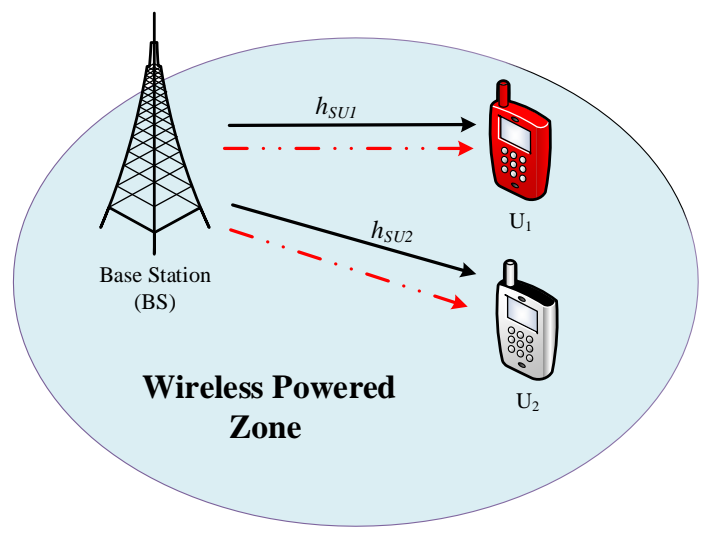

Figure 1. System model of NOMA with wireless power transfer

Considering such downlink, the signal received by the users is expressed as

$$
Y_{D L \rightarrow U_{i}}^{N M}=\sqrt{P_{D L}}\left(x_{U_{1}} a_{U_{1}}+x_{U_{2}} a_{U_{2}}\right) \frac{\left|h_{S i}\right|}{\sqrt{d_{i}^{l_{e}}}}+n_{i}
$$

where $P_{D L}$ is power to perform the downlink transmission for $i$ th user. $n_{i} \sim C N\left(0, \sigma_{i}^{2}\right)$ denotes AWGN at the $i$ th user with variance $\sigma_{i}^{2}$ and $P_{D L}=P_{S}\left(1-\beta_{e}\right)$. Requiring SIC at user $U_{1}$, the signal to noise ratio (SNR) at $U_{1}$ in such a novel scheme to detect $x_{U_{1}}$ is given by

$$
\gamma_{U_{1}, x_{U_{1}}}^{N M}=\frac{\left|h_{S U_{1}}\right|^{2} \rho_{U_{1}}}{d_{U_{1}}^{l_{e}}}
$$

where $\rho_{i}=\frac{P_{D L} a_{i}^{2}}{\sigma_{i}^{2}}$.

In NOMA, various users have various priorities in terms of the signal detection. Similarly, the signal to interference plus noise (SINR) at $U_{2}$ can be expressed as: 


$$
\gamma_{U_{2}, x_{U_{2}}}^{N M}=\frac{\left|h_{S U_{2}}\right|^{2} \rho_{U_{2}}}{\rho_{U_{1}}\left|h_{S U_{2}}\right|^{2}+d_{U_{2}}^{l_{e}}}
$$

\section{PERFORMANCE ANALYSIS}

\subsection{Channel statistic distribution}

The probability density function (PDF) and cumulative distribution function (CDF) of their gains follow gamma distributions formulated as respectively

$$
\begin{gathered}
f_{\left|h_{i}\right|^{2}}(x)=\frac{x^{m_{i}-1} e^{-\frac{x}{\Omega_{i}}}}{\Gamma\left(m_{i}\right) \Omega_{i}^{m_{i}}} \\
F_{\left|h_{i}\right|^{2}}(x)=1-\frac{\Gamma\left(m_{i}, \frac{x}{\Omega_{i}}\right)}{\Gamma\left(m_{i}\right)} \\
=1-e^{-\frac{x}{\Omega_{i}}} \sum_{i=0}^{m_{i}-1} \frac{1}{i !}\left(\frac{x}{\Omega_{i}}\right)^{i}
\end{gathered}
$$

where $\Omega_{i}=\frac{\lambda_{i}}{m_{i}}, \lambda_{i}$ is the fading severity factor and $m_{i}$ is the mean.

To evaluate the system performance once energy harvesting and feedback information for power allocation factors have been combined, we consider a metric, namely the ergodic rate. In particular, downlink is the main transmission which needs be investigated in term of the ergodic rate of the system in both NOMA and OMA schemes. This consideration is evaluated over a system model using an independent Rayleigh fading channel. In principle, the ergodic rate of each user is computed once the received SNR at corresponding $i$ th node has been established. It should be noted that during allocated time $\alpha_{d}$, the capacity of such communication can be evaluated.

\subsection{Ergodic rate performance in NOMA scenario}

In the NOMA mode, two users provide a total ergodic rate. The average ergodic rate in this mode can be expressed as [24]

$$
R^{N M}=\alpha_{d} \sum_{i} R_{i}^{N M}
$$

where $R_{i}^{N M}$ denotes the ergodic rate of two signals achieved by $i$ th NOMA users.

In principle, the instantaneous rate can be written as $R_{i}^{N M}=\log _{2}\left(1+\gamma_{U_{i}, x_{i}}^{N M}\right)$. It should be noted that $R_{U_{1}}^{N M}$ can be computed as

$$
\begin{aligned}
& R_{U_{1}}^{N M}=\log _{2}\left(1+\gamma_{U_{1}, x_{U_{1}}}^{N M}\right) \\
& =\frac{1}{\ln 2} \int_{0}^{\infty} \frac{1-F_{\gamma_{U_{1}, x_{1}}^{N M}}(x)}{1+x} d x
\end{aligned}
$$

Next, the PDF of $\gamma_{U_{1}, x_{U_{1}}}^{N M}$ can be express as

$$
\begin{aligned}
F_{\gamma_{U_{1}, x_{1}}^{N M}}(x) & =\operatorname{Pr}\left(\left|h_{S U_{1}}\right|^{2}<\frac{x d_{U_{1}}^{l_{e}}}{\rho_{U_{1}}}\right) \\
& =1-e^{-\frac{x}{\Omega_{U_{1}} \rho_{U_{1}}}} \sum_{i_{1}=0}^{m_{U_{1}}-1}\left(\frac{d_{U_{1}}^{l_{e}}}{\Omega_{U_{1}} \rho_{U_{1}}}\right)^{i_{1}} \frac{x^{i_{1}}}{i_{1} !}
\end{aligned}
$$


Next, we replace (10) into (9) and using the results from [25, 3.353.5], the following equation comes up

$$
\begin{aligned}
R_{U_{1}}^{N M} & =\frac{1}{\ln 2} \sum_{i_{1}=0}^{m_{U_{1}}-1} \frac{(-1)^{i_{1}-1}}{i_{1} !}\left(\frac{d_{U_{1}}^{l_{e}}}{\Omega_{U_{1}} \rho_{U_{1}}}\right)^{i_{1}} \\
& \times\left(e^{\frac{d_{U_{1}}^{\Omega_{U_{1}} \rho_{U_{1}}}}{2}} E i\left(-\frac{d_{U_{1}}^{l_{e}}}{\Omega_{U_{1}} \rho_{U_{1}}}\right)+\sum_{k_{1}=1}^{i_{1}}\left(k_{1}-1\right) !(-1)^{1-k_{1}}\left(\frac{d_{U_{1}}^{l_{e}}}{\Omega_{U_{1}} \rho_{U_{1}}}\right)^{-k_{1}}\right)
\end{aligned}
$$

where $E i(x)$ denotes as the exponential integral, it can be found in [25, 8.2.11.1]. In this model, $x_{U_{2}}$ is allocated higher power than $x_{U_{1}}$ in the superimposed NOMA signal. Therefore, the rate $R_{U_{2}}^{N M}$ can be given by

$$
\begin{aligned}
R_{U_{2}}^{N M} & =\log _{2}\left(1+\gamma_{U_{2}, x_{U_{2}}}\right) \\
& =\frac{1}{\ln 2} \int_{0}^{\infty} \frac{1-F_{Z_{1}}\left(x_{1}\right)}{1+x_{1}} d x-\frac{1}{\ln 2} \int_{0}^{\infty} \frac{1-F_{Z_{2}}\left(x_{2}\right)}{1+x_{2}} d x
\end{aligned}
$$

where $Z_{1}=\frac{\rho_{U_{2}}\left|h_{S U_{2}}\right|^{2}}{a_{2} d_{U_{2}}^{l e}}, Z_{2}=\frac{\rho_{U_{1}}\left|h_{S U_{2}}\right|^{2}}{d_{U_{2}}^{l e}}$. Then, it can be shown that

$$
\begin{aligned}
& F_{Z_{1}}\left(x_{1}\right)=1-e^{-\frac{x_{1} a_{2} d_{U_{2}}^{l} \Omega_{U_{2}} \rho_{U_{2}}}{m_{U_{2}}}} \sum_{i_{2}=0}^{m_{U_{2}}} \frac{x 1^{i_{2}}}{i_{2} !}\left(\frac{a_{2} d_{U_{2}}^{l_{e}}}{\Omega_{U_{2}} \rho_{U_{2}}}\right)^{i_{2}} \\
& F_{Z_{2}}\left(x_{2}\right)=1-e^{-\frac{x_{2} d_{U_{2}}^{l_{e}}}{\Omega_{U_{2}} \rho_{U_{1}}}} \sum_{i_{2}=0}^{m_{U_{2}}-1} \frac{x_{2}^{i_{2}}}{i_{2} !}\left(\frac{d_{U_{2}}^{l_{e}}}{\Omega_{U_{1}} \rho_{U_{1}}}\right)^{i_{2}}
\end{aligned}
$$

Similarly, we can obtain the closed-form of $R_{U_{2}}^{N M}$ as

$$
\begin{aligned}
& R_{U_{2}}^{N M}=\frac{1}{\ln 2} \sum_{i_{2}=0}^{m_{U_{2}}-1} \frac{(-1)^{i_{2}-1}}{i_{2} !}\left(\frac{d_{U_{2}}^{l_{e}}}{\Omega_{U_{2}} \rho_{U_{1}}}\right)^{i_{2}} \\
& \times\left(\left(a_{2}\right)^{i_{2}} e^{\frac{d_{U_{2} a_{2}}^{\Omega_{U_{2}} \rho_{2}}}{2}} E i\left(-\frac{d_{U_{2}}^{l_{e}} a_{2}}{\Omega_{U_{2}} \rho_{U_{2}}}\right)+\left(a_{2}\right)^{i_{2}} \sum_{k_{2}=1}^{i_{2}}\left(k_{2}-1\right) !(-1)^{1-k_{2}}\left(\frac{d_{U_{2}}^{l_{e}} a_{2}}{\Omega_{U_{2}} \rho_{U_{2}}}\right)^{-k_{2}}\right. \\
& \left.-e^{\frac{d_{U_{2}}^{\Omega_{e}}}{\Omega_{U_{2}} \rho_{1}}} E i\left(-\frac{d_{U_{2}}^{l_{e}}}{\Omega_{U_{2}} \rho_{U_{1}}}\right)-\sum_{k_{2}=1}^{i_{2}}\left(k_{2}-1\right) !(-1)^{1-k_{2}}\left(\frac{d_{U_{2}}^{l_{e}}}{\Omega_{U_{2}} \rho_{U_{1}}}\right)^{-k_{2}}\right)
\end{aligned}
$$

Finally, putting (9) and (13) into (6). We can obtain the closed-form expression of sum ergodic rare for considered system $R^{N M}$ as

$$
\begin{aligned}
& R^{N M}=\frac{\alpha_{d}}{\ln 2}\left(\sum _ { i _ { 1 } = 0 } ^ { m _ { U _ { 1 } } - 1 } \frac { ( - 1 ) ^ { i _ { 1 } - 1 } } { i _ { 1 } ! } ( \frac { d _ { U _ { 1 } } ^ { l _ { e } } } { \Omega _ { U _ { 1 } } \rho _ { U _ { 1 } } } ) ^ { i _ { 1 } } \left(e^{\frac{d_{U_{1}}^{l_{e}}}{\Omega_{U_{1}} \rho_{U_{1}}}} E i\left(-\frac{d_{U_{1}}^{l_{e}}}{\Omega_{U_{1}} \rho_{U_{1}}}\right)\right.\right.
\end{aligned}
$$

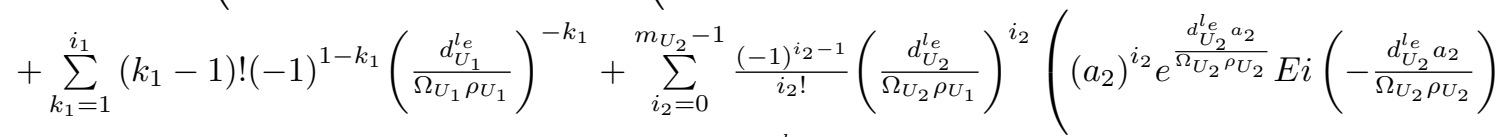

$$
\begin{aligned}
& +\left(a_{2}\right)^{i_{2}} \sum_{k_{2}=1}^{i_{2}}\left(k_{2}-1\right) !(-1)^{1-k_{2}}\left(\frac{d_{U_{2}}^{l_{e}} a_{2}}{\Omega_{U_{2}} \rho_{U_{2}}}\right)^{-k_{2}}-e^{\frac{d_{U_{2}}^{l_{e}}}{\Omega_{U_{2}} \rho_{U_{1}}}} E i\left(-\frac{d_{U_{2}}^{l_{e}}}{\Omega_{U_{2}} \rho_{U_{1}}}\right) \\
& -\sum_{k_{2}=1}^{i_{2}}\left(k_{2}-1\right) !(-1)^{1-k_{2}}\left(\frac{d_{U_{2}}^{l_{e}}}{\Omega_{U_{2}} \rho_{U_{1}}}\right)^{-k_{2}}
\end{aligned}
$$




\section{NUMERICAL AND SIMULATION RESULTS}

This section provides the numerical results demonstrating the ergodic rate performance of the proposed NOMA. More importantly, the results have proven the corroboration of the analytic results. In these simulations, a fair agreement can be raised between the simulated and analytic lines in all of the results. As in many other sources, the distances between the pair of nodes are normalized. For these power allocation factors received in the first time slot of the NOMA case, $l_{e}=2, a_{U_{1}}^{2}=0.2, a_{U_{2}}^{2}=0.8, \alpha_{d}=0.5, \beta_{e}=0.1$, $=\Omega_{U_{1}}=\Omega_{U_{2}}=1$ and $m_{U_{1}}=m_{U_{2}}=2$ has been set, except for specific cases. In addition, the distance is set as $d_{U_{1}}=5, d_{U_{2}}=10$.

Figure 2 depicts ergodic rate of considered system versus SNR. Increasing SNR, ergodic rate can be improved significantly, especially in the high SNR regime. As $\beta_{e}=0.2$, that means more power is employed to signal processing, therefore higher ergodic rate can be obtained.

It can observed in Figure 3 that varying parameter $m$ of Nakagami- $m$ fading results in slight varying in ergodic performance. It is confirmed that although energy constraint at two users, sum ergodic capacity is still guaranteed.

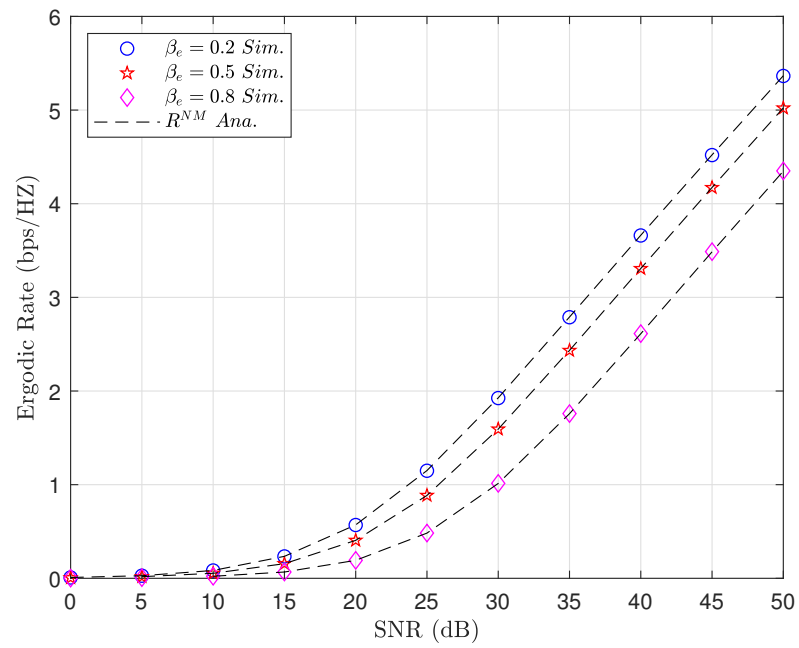

Figure 2. Ergodic capacity performance versus SNR

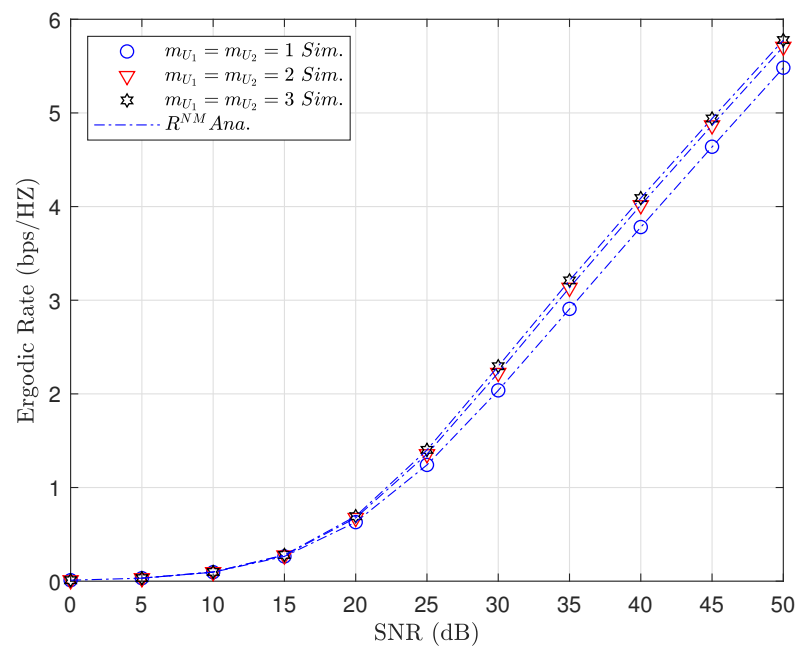

Figure 3. Ergodic capacity performance versus SNR 


\section{CONCLUSION}

In this study, the energy harvesting policy provides more opportunities to prolong the lifetime of the operation of users in considered NOMA scheme. As regards the individual user's ergodic capacity in NOMA, the exact expression of the ergodic rate has been described, and relevant results verified in numerical simulations. The sum ergodic capacity performance were evaluated in numerical results. The impact of required SNR, power splitting factor in energy harvesting protocol were jointly examined in many situations to illustrate the advantages of NOMA performance while satisfying the fairness in NOMA users. Finally, wireless power transfer still provide reasonable performance and this technique is recommended to be applied both WPT and NOMA for $5 \mathrm{G}$ wireless deployment.

\section{REFERENCES}

[1] Y. Xu, C. Shen, Z. Ding, X. Sun, S. Yan, and G. Zhu, "Joint Beamforming Design and Power Splitting Control in Cooperative SWIPT NOMA Systems," accepted by IEEE ICC., vol. 65, pp. 18, pp. 4874-4886, May 2017.

[2] Y. Saito, Y. Kishiyama, A. Benjebbour, T. Nakamura, A. Li, and K. Higuchi, "Non-Orthogonal Multiple Access (NOMA) for Cellular Future Radio Access," in Proc. IEEE Veh. Technol. Conf, pp. 1-5, 2013.

[3] Z. Ding, Y. Liu, J. Choi, Q. Sun, M. Elkashlan, C. L. I, and H. V. Poor, ”Application of Non-Orthogonal Multiple Access in LTE and 5G Networks," IEEE Commun. Mag., vol. 55, no. 2, pp. 185-191, Feb. 2017.

[4] D. Tse, P. Viswanath. "Fundamentals of Wireless Communication." UK: Cambridge Univ. Press, 2005.

[5] Z. Ding, Z. Yang, P. Fan, and H. V. Poor, "On the Performance of NonOrthogonal Multiple Access in 5G Systems with Randomly Deployed Users," IEEE Signal Process. Lett., vol. 21, no. 12, pp. 1501-1505, Dec. 2014.

[6] Z. Ding, P. Fan, and H. V. Poor, "Impact of User Pairing on 5G Nonorthogonal Multiple-Access Downlink Transmissions," IEEE Trans. Veh. Tech., vol. 65, no. 8, pp. 6010-6023, Aug. 2016.

[7] F. De Rango, M. Tropea, "Swarm intelligence based energy saving and load balancing in wireless ad hoc networks," in Proc. of the 2009 Workshop on Bio-inspired Algorithms for Distributed Systems, BADS '09, pp. 77-83, 2009.

[8] F. De Rango, M. Tropea, "Energy saving and load balancing in wireless ad hoc networks through ant-based routing," in Proc. of the International Symposium on Performance Evaluation of Computer and Telecommunication Systems 2009, SPECTS 2009., pp. 117-124, 2009.

[9] Dinh-Thuan Do, H.-S. Nguyen, M Voznak, T.-S. Nguyen, "Wireless powered relaying networks under imperfect channel state information: system performance and optimal policy for instantaneous rate," Radioengineering, no. 26, pp. 869-877, 2017.

[10] X.-X. Nguyen, Dinh-Thuan Do, "Optimal power allocation and throughput performance of full-duplex DF relaying networks with wireless power transfer-aware channel," EURASIP Journal on Wireless Communications and Networking, no. 1, pp. 152, 2017.

[11] K. T. Nguyen, Dinh-Thuan Do, X. X. Nguyen, N. T. Nguyen, D. H. Ha, "Wireless information and power transfer for full duplex relaying networks: performance analysis," in Proc. of Recent Advances in Electrical Engineering and Related Sciences (AETA 2015), HCMC, Vietnam, pp. 53-62, 2015.

[12] T.-L. Nguyen, Dinh-Thuan Do, "A new look at AF two-way relaying networks: energy harvesting architecture and impact of co-channel interference," Annals of Telecommunications, Vol. 72, No. 11, pp. 669-678, 2017.

[13] Dinh-Thuan Do, H.-S. Nguyen, "A Tractable Approach to Analyze the Energy-Aware Two-way Relaying Networks in Presence of Co-channel Interference," EURASIP Journal on Wireless Communications and Networking, 2016.

[14] Y. Liu, Z. Ding, M. Elkashlan, and H. V. Poor, "Cooperative NonOrthogonal Multiple Access with Simultaneous Wireless Information and Power Transfer," IEEE J. Sel. Areas Commun., vol. 34, no. 4, pp.938-953, April 2016.

[15] Dinh-Thuan Do and C.-B. Le, "Application of NOMA in Wireless System with Wireless Power Transfer Scheme: Outage and Ergodic Capacity Performance Analysis," Sensors, vol. 18, no. 10, p.3501, 2018.

[16] Dinh-Thuan Do, M.-S. Van Nguyen, T.-A. Hoang and M Voznak, "NOMA-Assisted Multiple Access Scheme for IoT Deployment: Relay Selection Model and Secrecy Performance Improvement," Sensors, vol. 19, no. 3, pp. 736, 2019.

[17] Vaezi, Mojtaba, Zhiguo Ding, and H. Vincent Poor. "Multiple access techniques for 5G wireless networks and beyond," Springer, 2019.

[18] H. Chingoska, Z. Hadzi-Velkov, I. Nikoloska, and N. Zlatanov, "Resource allocation in wireless powered communication networks with nonorthogonal multiple access," IEEE Wireless Commun. Lett., vol. 5, no. 6, pp. 684-687, Dec. 2016.

[19] Y. Liu, Z. Ding, M. Elkashlan, and H. V. Poor, "Cooperative nonorthogonal multiple access with simultaneous wireless information and power transfer," IEEE J. Sel. Areas Commun., vol. 34, no. 4, pp. 938-953, Apr. 2016.

[20] T.-L. Nguyen and Dinh-Thuan Do, "Exploiting Impacts of Intercell Interference on SWIPT-assisted Non-orthogonal Multiple Access," Wireless Communications and Mobile Computing, vol. 2018, Article ID 2525492, 12 pages , 2018.

[21] D-T. Do et al., "Wireless power transfer enabled NOMA relay systems: two SIC modes and performance evaluation," TELKOMNIKA (Telecommunication, Computing, Electronics and Control) , vol. 17, no.6, pp. 2697-2703, 2019. 
[22] Dinh-Thuan Do and T.-T. Thi Nguyen, "Exact Outage Performance Analysis of Amplify-and Forward-Aware Cooperative NOMA," TELKOMNIKA (Telecommunication, Computing, Electronics and Control), vol. 16 no. 5, pp. 1966-1973, 2018.

[23] Dinh-Thuan Do, Chi-Bao Le and A.-T. Le, "Cooperative underlay cognitive radio assisted NOMA: secondary network improvement and outage performance," TELKOMNIKA (Telecommunication, Computing, Electronics and Control), vol. 17, no. 5, pp. 2147-2154, 2019

[24] S. K. Zaidi, S. F. Hasan and X. Gui, "Evaluating the Ergodic Rate in SWIPT-Aided Hybrid NOMA," IEEE Communications Letters, vol. 22, no. 9, pp. 1870-1873, 2018

[25] D. Zwillinger. "Table of Integrals, Series, and Products, 7 Ed.," Academic Press, 2007. 